\title{
Overexpression of OsOSM1 Enhances Resistance to Rice Sheath Blight
}

X. Xue, Z. X. Cao, X. T. Zhang, Y. Wang, Y. F. Zhang, Z. X. Chen, X. B. Pan, and S. M. Zuo, Jiangsu Key Laboratory of Crop Genetics and Physiology/Co-Innovation Center for Modern Production Technology of Grain Crops, Key Laboratory of Plant Functional Genomics of the Ministry of Education, Yangzhou University, Yangzhou 225009, China

\begin{abstract}
Xue, X., Cao, Z. X., Zhang, X. T., Wang, Y., Zhang, Y. F., Chen, Z. X., Pan, X. B., and Zuo, S. M. 2016. Overexpression of OsOSM1 enhances resistance to rice sheath blight. Plant Dis. 100:1634-1642.

Sheath blight (SB), caused by Rhizoctonia solani, is one of the most destructive rice diseases worldwide. It has been difficult to generate SBresistant varieties through conventional breeding because of the quantitative nature of rice resistance to SB. In this study, we found that overexpression of the OSOSM1 gene, encoding an osmotin protein belonging to the pathogenesis-related protein 5 family, is able to improve rice resistance to SB in field tests. Although there are two osmotin genes in rice, OsOSM1 is the one mainly expressed in leaf sheath at the booting stage, coinciding with the critical stage of SB development in the field. In addition, OsOSM1 expression is strongly induced by $R$. solani in SBresistant rice variety YSBR1 but not in susceptible varieties, suggesting its involvement in SB resistance. Overexpression of OsOSM1 (OsOSM1ox) in susceptible variety Xudao 3 significantly increases resistance to SB in

transgenic rice. The OsOSM1 mRNA levels in different transgenic lines are found to be positively correlated with their SB resistance levels. Intriguingly, although extremely high levels of OSOSM1 were detrimental to rice development, appropriately elevated levels of OsSOM1 were obtained that enhanced rice SB resistance without affecting rice development or grain yield. The OsSOM1 protein is localized on plasma membrane. OsOSM1 is upregulated by jasmonic acid (JA); furthermore, JA-responsive marker genes are induced in OsOSM1ox lines. These results suggest that the activation of JA signaling pathway may account for the increased resistance in transgenic OSOSM1ox lines. Taken together, our results demonstrate that OSOSM1 plays an important role in defense against rice SB disease and provides a new target for engineering resistance to $\mathrm{SB}$.
\end{abstract}

Sheath blight (SB) is one of the most destructive rice diseases worldwide, causing severe losses in rice production and reduction in grain quality by infecting and destroying the rice sheath and leaves under favorable conditions (Lee and Rush 1983). Due to the widespread cultivation of semidwarf, high nitrogen-responsive, and high-yield rice varieties, the SB disease has become increasingly serious and, in some regions, it has become the most serious disease (Kagale et al. 2011). Its causal agent, Rhizoctonia solani Kühn, is a soilborne fungus with strong saprophytism and a broad host range. Although chemical control is an effective method to control this fungus, it is unsustainable because of high costs and serious environmental pollution.

Planting of resistant cultivars is the most economic, effective, and environmentally friendly way to manage SB. However, it has proven very difficult to develop SB-resistant varieties by conventional breeding because of lack of completely resistant germplasms (Chen et al. 2014). All known resistant germplasms confer only partial resistance to SB and are controlled by multiple genes or quantitative trait loci (QTL). To date, approximately $50 \mathrm{SB}$ resistance QTL (SBR QTL) were mapped residing on all 12 rice chromosomes in various germplasms, including cultivated varieties, deep-water varieties, and wild species (Chen et al. 2014; Jia et al. 2009; Liu et al. 2013; Srinivasachary et al. 2011; Xu et al. 2011; Zuo et al. 2006, 2010). A few studies have shown that introgression of SBR QTL by marker-assisted selection is a feasible approach to improve rice SB resistance quantitatively (Chen et al. 2014). However, the complex interactions between QTL and genetic backgrounds have also been

Corresponding authors: S. M. Zuo; E-mail: smzuo@yzu.educn; and X. B. Pan; E-mail: shuidao@yzu.edu.cn

*The $\boldsymbol{e}$-Xtra logo stands for "electronic extra" and indicates that one supplementary figure and one supplementary table are published online.

Accepted for publication 2 March 2016.

http://dx.doi.org/10.1094/PDIS-11-15-1372-RE

(c) 2016 The American Phytopathological Society observed (Chen et al. 2014; Yin 2008). Consequently, not all SBR QTL of interest function in different rice varieties, complicating QTL pyramiding and utilization of these SBR QTL in breeding practice.

As a promising technology for crop improvement, the transgenic approach has been widely employed to develop crops resistant to either pests or pathogens (Kok et al. 2014; Wang et al. 2015). Transgenic rice plants with pathogen-inducible ethylene (ET) production by manipulating the OsACS2 (1-aminocyclopropane-1-carboxylic acid synthase, a key enzyme of ET biosynthesis) gene exhibit broad-spectrum disease resistance to both fungal pathogens Magnaporthe oryzae and $R$. solani (Helliwell et al. 2013). Remarkably, OsACS2 transgenic rice lines display no inferior alterations on important agronomical traits compared with the wild type (WT), indicating its potential in developing transgenic rice with SB resistance. Polygalacturonase-inhibiting proteins (PGIP) are believed to be effective on inhibiting polygalacturonase activity, one of the critical pathogenic factors secreted by necrotrophic pathogens such as $R$. solani (De Lorenzo et al. 2001). Two studies have confirmed that overexpressing OSPGIP1 could enhance rice resistance to the SB disease and had almost no effects on rice development (Chen et al. 2015; Wang et al. 2015). In addition to these genes, most others that were confirmed to improve SB resistance belong to pathogenesis-related (PR) protein families. For instance, transgenic rice constitutively expressing infection-related rice gene chill (encoding chitinase, PR-3) showed significantly enhanced resistance to SB (Datta et al. 2001); similarly, rice coexpressing tlp-D34 (encoding thaumatinlike protein, PR-5) and chill (Shah et al. 2013) displayed enhanced resistance to SB. Coexpression of $\mathrm{RCH} 10$ (encoding chitinase, PR-3) and AGLU1 (encoding alfalfa $\beta$-1,3-glucanase, PR-2) in rice conferred high levels of resistance to SB (Mao et al. 2014). Together, these results demonstrate that modification of the expression levels of rice genes can significantly enhance rice resistance to SB.

Osmotin and osmotin-like proteins (OLP) belong to thaumatin-like proteins (TLP) of the PR-5 family because they all contain a typical thaumatin domain. Osmotin is originally regarded as a salt-induced protein in osmotically stressed tobacco (Nicotiana tabacum L.) cells (Singh et al. 1987); it is involved in plant permeability stress and defense responses because of its antibacterial properties in vivo against a broad range of 
plant pathogens (Narasimhan et al. 2005). In contrast to most other plant PR-5 proteins, osmotin and OLP are basic isoforms and, presumably, accumulate in the vacuoles of plant cells instead of extracellularly (Anžlovar and Dermastia 2003). Osmotin overexpression in potato

Table 1. Primers for gene transcription analysis used in this study

\begin{tabular}{llc}
\hline Name & \multicolumn{1}{c}{ Primer sequence $\left(\mathbf{5}^{\prime} \mathbf{- 3}^{\prime}\right.$ ) } & $\begin{array}{c}\text { Product } \\
\text { size/bp }\end{array}$ \\
\hline Actin-q-F & CTTCATAGGAATGGAAGCTGCGGGTA & 197 \\
Actin-q-R & CGACCACCTTGATCTTCATGCTGCTA & $\ldots$ \\
OsOSM1-q-F & CCGACAACTAGCTAGCCTA & 207 \\
OsOSM1-q-R & CGCACGTACAAACATAAGG & $\ldots$ \\
PAL1-F & AGCACATCTTGGAGGGAAGCT & 120 \\
PAL1-R & GCGCGGATAACCTCAATTTG & $\ldots$ \\
PR10-F & CCCTGCCGAATACGCCTAA & 120 \\
PR10-R & CTCAAACGCCACGAGAATTTG & $\ldots$ \\
COI1-F & GGGGTGATGATGATCCAG & 188 \\
COI1-R & AAGTAGCACAAGCCGAAA & $\ldots$ \\
\hline
\end{tabular}

significantly delays development of late blight disease symptoms (Liu et al. 1994). Pepper osmotin-like protein 1 ( $\mathrm{CaOLP1}$ ) plays a critical role in defense response, cell death, and oxidative burst (Choi et al. 2013). However, whether osmotin proteins are able to improve rice $\mathrm{SB}$ resistance remains unclear.

In this study, we identified two osmotin genes in the rice genome. OsOSM1 is highly expressed in leaf sheaths at the booting stage, coinciding with where and when the SB fungus mainly infects rice. Moreover, OsOSMI expression is significantly induced by $R$. solani in the SB-resistant variety YSBR1 but not in susceptible varieties Lemont or Xudao 3. Proper, elevated levels of OSOSM1 in Xudao 3 (XD3) rice enhances resistance to $\mathrm{SB}$ without affecting rice development or grain yield. We also show that OsOSM1 functions in jasmonic acid (JA) signaling pathway and hypothesize that the activation of JA defense-related genes is responsible for the enhanced resistance.

\section{Materials and Methods}

Phylogenetic analysis. The OsOSM1 (LOC_Os12g38170) protein sequence was obtained from the Rice Genome Annotation Project

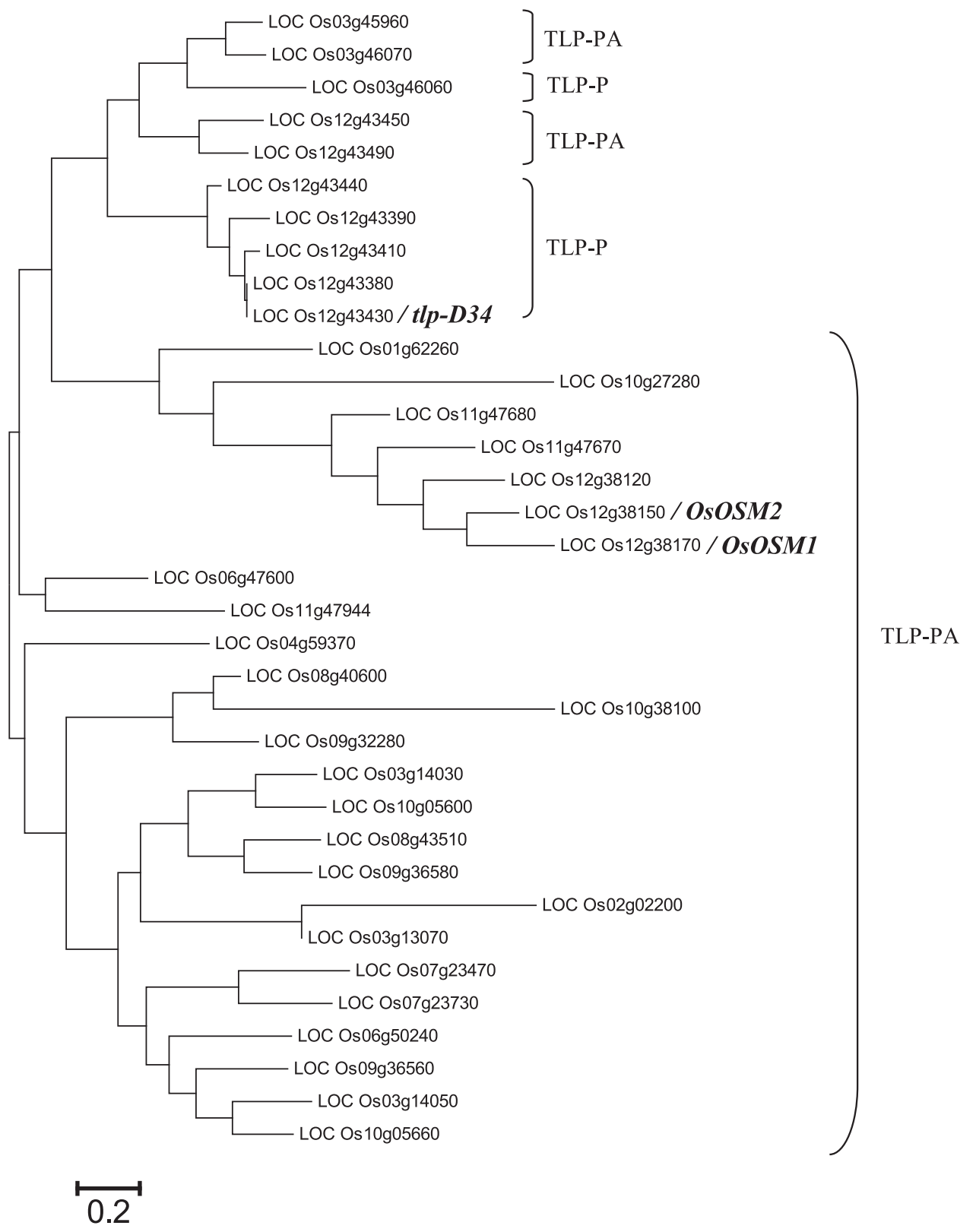

Fig. 1. Phylogenetic trees of 35 thaumatin-like protein (TLP) domain-containing rice genes. The rice TLP protein family contains the TLP-PA and TLP-P subfamilies. The tlp-D34 gene was the only rice TLP-domain-containing protein reported to confer resistance to rice sheath blight (Shah et al. 2013). The two osmotin genes (OsOSM1 and OsOSM2) are clustered together. The tree was constructed using the maximum-likelihood method with default settings. The gap/missing data were treated by partial deletion with site coverage cut off at $90 \%$. 
(http://rice.plantbiology.msu.edu/). The domains of the OsOSM1 protein were predicted using the SMART program (http://smart. embl-heidelberg.de/) (Schultz et al. 1998). The core domain sequence was then used to search the rice genome to identify homologs on the National Center for Biotechnology Information (NCBI) using BLASTp (http://blast.ncbi.nlm.nih.gov/Blast.cgi). The entire phylogenetic analysis for homologs was performed in the MEGA 6 software (Tamura et al. 2013). The trees were constructed by the Maximum Likelihood method with default settings. The gap/missing data were treated by partial deletion with $90 \%$ site coverage cut off. The phylogeny was tested using Boots Trapping with one thousand replications.

Plant materials and growth conditions. Lemont and XD3 are japonica rice cultivars from Louisiana in the United States and Jiangsu, China, respectively, that are susceptible to rice SB. YSBR1 is an indica rice line from Yangzhou, China, that shows high resistance to SB (Zuo et al. 2009). All rice seed, including OsOSM1-overexpressing (OsOSM1ox) rice lines in $\mathrm{XD} 3$ background, were germinated at $32^{\circ} \mathrm{C}$ for 2 days, then grown in the field or in the greenhouse. In
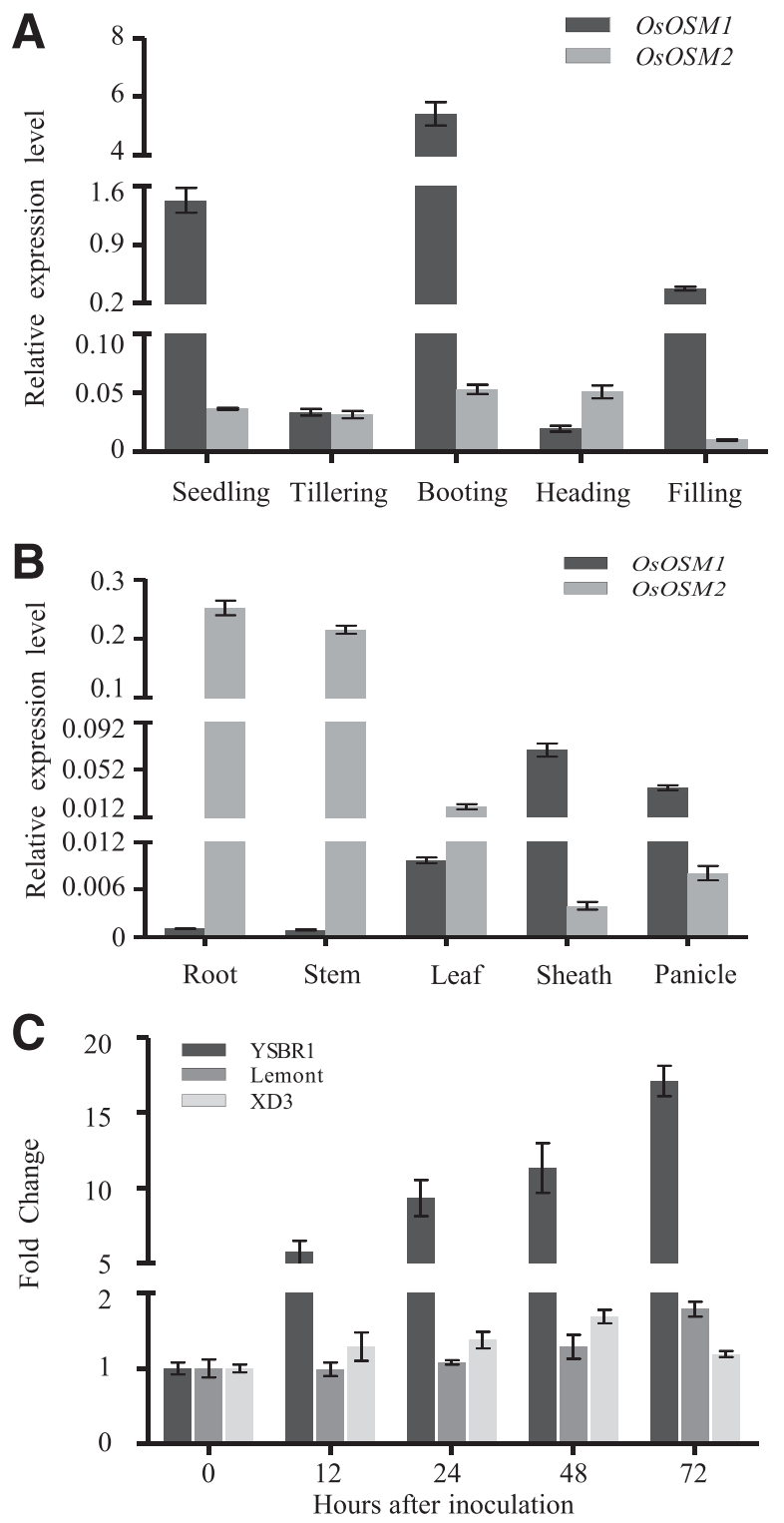

Fig. 2. Expression patterns of the two rice osmotin genes. A, Expression levels of the two osmotin genes (LOC_Os12g38170/OsOSM1, LOC_Os12g38150/OsOSM2) at different developmental stages of rice. B, Expression patterns of the two osmotin genes in different organs at the heading stage. C, Induction of OsOSM1 and OsOSM2 expressions in response to Rhizoctonia solani infection in 4-week-old rice seedlings of different rice varieties. Total RNA was extracted from the same amount rice tissues. The $\beta$-actin gene was used as the internal control. Each bar represents the average and standard deviation of at least three replicates. the field, each rice line was planted in a plot containing three rows, with 12 plants/row. The distances between plants and between rows were 17 and $22 \mathrm{~cm}$, respectively. In the greenhouse, the seedlings were planted in pots (12 $\mathrm{cm}$ in diameter) and grown under a 14-h photoperiod daily at $28^{\circ} \mathrm{C}$. Each pot contained five plants.

Pathogen inoculation. For SB fungus inoculation, the $Y N-7$ isolate with moderate pathogenicity was used. This strain has been used in several previous studies (Chen et al. 2015; Zhu et al. 2014; Zuo et al. 2008). Briefly, autoclaved, truncated, thin matchsticks with a length of 0.8 to $1.0 \mathrm{~cm}$ were incubated with $Y N-7$ on potato dextrose broth medium for 3 to 5 days in darkness at $28^{\circ} \mathrm{C}$ (Zou et al. 2000). Then, the woody matchsticks colonized by the SB fungus were used as the inoculum.

For seedling inoculation assays in the greenhouse, the inoculum was put at the base of each 4-week-old rice seedling. The completely randomized experimental design with three replications was used in seedling inoculation assay. For each rice line, each replicate contained at least eight individuals from two pots. After inoculation, all plants were covered with a plastic film to form a moist chamber. The humidity and temperature in the moist chamber ranged from 85 to $95 \%$ and 25 to $31^{\circ} \mathrm{C}$, respectively. Leaf sheath samples were harvested at $0,12,24,48$, and $72 \mathrm{~h}$ after inoculation for total RNA extraction.

For adult inoculation assays in the field, the inoculation was conducted at the late-tillering stage of rice development (Zuo et al. 2013). Each rice line was planted in three plots (replicates). In each plot, rice lines were planted in three rows and the central 10 plants of the middle row were inoculated with the SB fungus. Using a nipper, the inoculum was placed inside the third sheath from top without changing the sheath-stem holding status. Because the second sheath from the top at this time does not elongate further, the inoculum stably maintains inside the third sheath. In order to increase successful inoculation rates, the main stem and the two largest tillers of each plant were inoculated. Disease severity was rated approximately 30 days after heading. A modified 0-to-9 disease scoring system was used in this study, where 0 is defined as no disease and 9 indicates that the plant is dead or collapsed (Zuo et al. 2013).

Treatment with defense response inducers. For the treatment of defense response-inducing molecules, 4-week-old rice YSBR1 seedlings were sprayed with $50 \mu \mathrm{M}$ kinetin (KT), $500 \mu \mathrm{M}$ ethephon, $100 \mu \mathrm{M}$ methyl jasmonate, $500 \mu \mathrm{M}$ salicylhydroxamic acid, and $8 \mathrm{mM}$ salicylic acid (SA) until liquid dripped off leaves (De Vleesschauwer 2008; De Vleesschauwer et al. 2010; Ito and Kurata 2006; Penninckx et al. 1996; Xie et al. 1998). Control plants were treated in the same way with diluted water containing respective dissolvents. For each treatment, at least eight individual plants were sprayed in a completely randomized experimental design and all experiments were repeated two times. Leaf samples were harvested $0,1,2,3,4$, and 5 days after treatment.

Tobacco transient expression and confocal microscopy. The OsOSM1 gene was joined with the C-terminal end of green fluorescent protein (GFP) to yield a fusion protein. The coding region of the target gene was amplified using the primers $5^{\prime}$-CCGCTCGAG ATGGCGAACAAGCTGCAGCTC-3' (forward, XhoI site underlined) and 5'-CGGGATCCCTAGTTGTCGGCGACGTCGAC-3' (reverse, BamHI site underlined), and the amplified coding region was inserted into the XhoI and BamHI sites of vector pGDG (Goodin et al. 2002); the resulting construct was used to transform Agrobacterium tumefaciens cells (EHA105). Intact leaves of 4-week-old tobacco ( $N$. benthamiana) plants were infiltrated with EHA105 cells harboring either pGDG or pGDG-OsOSM1. The expressed fusion protein was monitored 2 to 3 days after infiltration by confocal microscopy (LSM 710 Meta; Carl Zeiss). Fluorophores were excited using an argon laser at $488 \mathrm{~nm}$ (GFP), and bright-field images were collected using the transmitted light detector.

Plasmid construction and plant transformation. Full-length OsOSM1 cDNA was cloned from Nipponbare. The coding nucleotide sequence was amplified using primers 5'-CGGGATCCCGCA TCGCTGCCAAGTGGTT-3'(forward, BamHI site underlined), and 5'-GGGGTACCCCGACGGTGCGGATGAGCT-3' (reverse, KpnI site 

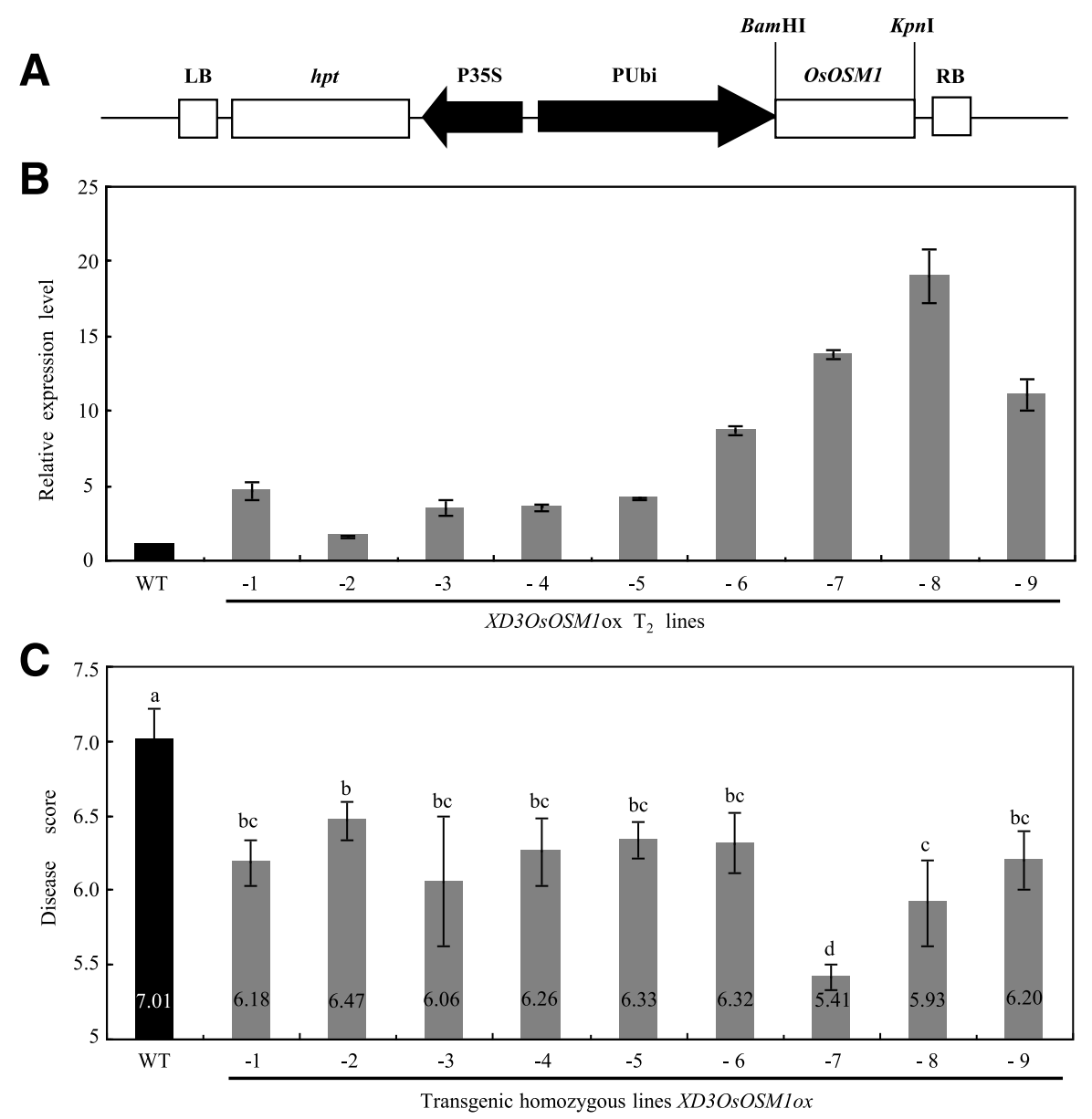

D
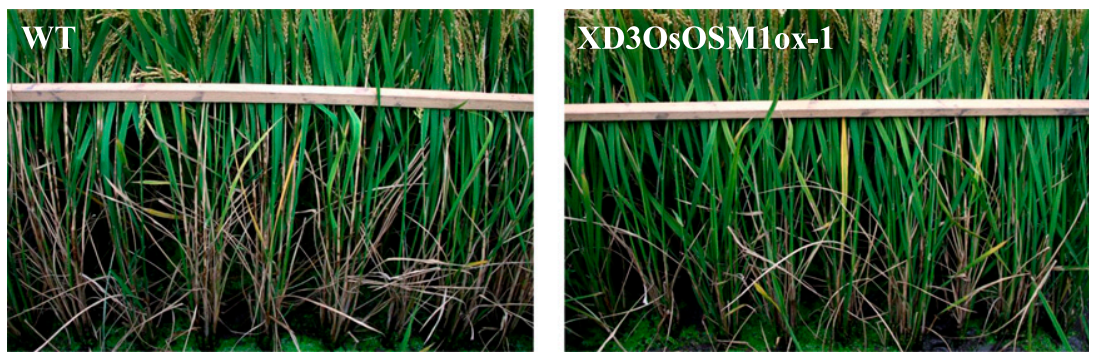

$\mathbf{E}$

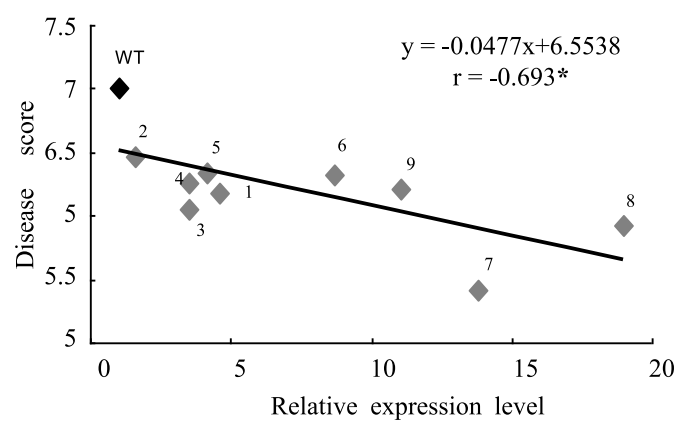

Fig. 3. Overexpression of OsOSM1 enhances rice resistance to sheath blight (SB). A, Structure of the T-DNA region of the OsOSM1ox construct. LB = left border, RB = right border, $\mathrm{Hpt}=$ hygromycin phosphotransferase, $\mathrm{P} 35 \mathrm{~S}=$ Cauliflower mosaic virus $35 \mathrm{~S}$ promotor, and Pubi = maize ubiquitin-1 promoter. B, Relative expression levels of OsOSM1 in wild-type (WT) and nine independent transgenic XD3 OsOSM10x lines. Total RNA was extracted from leaf sheath at the adult stage. The $\beta$-actin gene was used as the internal control. C, SB disease scores of WT and transgenic lines. The inoculation was conducted at the later tillering stage and disease scores were rated 30 days after heading using a 0-to-9 rating scale. D, Photographs of WT and transgenic line XD3 OsOSM10x-1 35 days postinoculation with the SB fungus in the field. E, Relationship between relative expression level of OSOSM1 and disease scores in independent transgenic lines. 
underlined). The amplified cDNA fragment was digested with BamHI and $K p n \mathrm{I}$ and then inserted into the BamHI and KpnI sites of vector pCAMBIA1301-Ubi for generating OsOSM1 overexpression construct pCAMBIA1301-Ubi-OsOSM1. Agrobacterium-mediated rice transformation was performed as described by Hiei et al. (1994).

Genomic DNA isolation and polymerase chain reaction analysis. Genomic DNA was isolated from leaves of 3- to 4-week-old plants. In order to confirm the presence of the OsOSM1ox transgene, polymerase chain reaction (PCR) was performed using genomic DNA as template and primers hpt-F (5'-ATTTGTGTACGCCCGACAGT-3') and hpt-R (5'-GGATATGTCCTGCGGGTAAA-3') specific to the hygromycin selection marker gene.

RNA extraction, reverse transcription, and quantitative reversetranscription PCR analysis. Total RNA was isolated using the TRIzol reagent according to the manufacturer's instructions (Invitrogen). RNA quantity, integrity, and purity were determined by agarose gel electrophoresis and Nano-Drop spectrophotometer. Total RNA $(2 \mu \mathrm{g})$ was then treated with RNase-free DNase I, and first-strand cDNA was synthesized by reverse transcription (RT) using an oligo (dT) primer (Roche). The rice actin gene was used as internal control for RT-PCR experiments. The amplification reactions were conducted using the SYBR PrimeScript RT-PCR Kit (TaKaRa) with gene-specific primers listed in Table 1. The threshold cycle was determined by using the maximum-second derivative function of the software for the Applied Biosystems 7900HT Real-Time PCR System. Each sample contains three biological replicates and each biological replicate includes three technical replicates.

Evaluation of agronomic traits. Agronomic traits were measured for transgenic plants and the WT control, which were planted in three rows per line in a field separated from the SB test field. Each row contained 12 plants. The distances between two plants and between rows and field management are the same as those in the SB test field. Agronomic traits, including heading date, tiller angles, plant height, flag leaf length, flag leaf width, and yield components of panicle length, panicle number per plant, seed number per panicle, ratio of filled grains, and 1,000-grain weight, were measured, as described previously (Zuo et al. 2013).
Statistical analysis. Data of SB disease severity and agronomic traits were analyzed using one-factor analysis of variance procedure in the SPSS12.05 program. Mean comparisons (least significant difference) were used to reveal the difference among rice lines tested for the SB disease severity, agronomic traits, and yield-related traits, where $P=$ 0.05 and $P=0.01$ were used to measure whether the differences were statistically significant and highly significant, respectively.

\section{Results}

Rice osmotin genes and phylogenetic analysis. We identified two osmotin genes, OsOSM1 (LOC_Os12g38170) and OsOSM2 (LOC_Os12g38150), in the rice genome by the Putative Function Search Tool (http://rice.plantbiology.msu.edu/analyses_search_ putative.shtml). Both genes contain a well-defined thaumatin domain identified using the SMART program and, therefore, are assigned to the TLP class of the PR-5 family. By using BLASTp tool in NCBI, we then identified another 33 proteins which all contain a typical thaumatin domain; they were not annotated as osmotin proteins but, rather, as thaumatin or TLP (Supplementary Table S1). TLP were generally classified into three subfamilies: TLP-P, TLP-PA, and TLP-F. The TLP-P subfamily includes six genes and one of them (LOC_Os12g43430/tlp-D34) has been shown to enhance rice SB resistance when overexpressed (Datta et al. 1999; Shah et al. 2013). In all, 29 genes, including OsOSM1, belong to the TLP-PA subfamily; no TLP-F genes were identified in the rice genome (Fig. 1). The OsOSM1 protein shares the highest identity with OsOSM2 on protein sequence according to the phylogenetic tree (Fig. 1). The roles of these remaining TLP, including the two osmotins, are unknown, except for gene LOC_Os12g43430/tlp-D34.

OsOSM1 expression is induced by $\mathrm{SB}$ infection in resistant rice cultivars. Generally, $R$. solani initially infects the rice sheath at the late tillering (booting) stage and develops quickly on rice sheaths in the field. Therefore, we set out to assess whether these two $O S O S M$ genes are expressed at the right time and place. We found that OsOSM1 has the highest expression level at the booting stage, followed by the seedling stage, whereas OsOSM2 displays low expression levels across all five stages tested (Fig. 2A). For their

Table 2. Major agronomic traits of different transgenic lines and the wild-type (WT) control XD3 \pm standard deviation $(\mathrm{SD})^{\mathrm{z}}$

\begin{tabular}{|c|c|c|c|c|c|}
\hline Line & HD \pm SD $($ doy $)$ & $\mathbf{T A} \pm \mathbf{S D}\left(^{\circ}\right)$ & $\mathrm{PH} \pm \mathrm{SD}(\mathrm{cm})$ & FLL \pm SD $(\mathbf{c m})$ & $\mathrm{FLW} \pm \mathrm{SD}(\mathrm{cm})$ \\
\hline WT & $96.33 \pm 0.53 \mathrm{ab}$ & $22.46 \pm 0.53 \mathrm{ab}$ & $84.88 \pm 0.40 \mathrm{a}$ & $20.79 \pm 0.34$ cde & $1.28 \pm 0.03 \mathrm{abc}$ \\
\hline XD3OSM1ox-1 & $95.50 \pm 0.58 b c$ & $24.29 \pm 2.38 \mathrm{a}$ & $85.13 \pm 0.72 \mathrm{a}$ & $20.92 \pm 0.84 \mathrm{cde}$ & $1.21 \pm 0.04 \mathrm{c}$ \\
\hline XD3OSM1ox-2 & $95.33 \pm 1.20 b c$ & $23.60 \pm 0.49 a b$ & $84.08 \pm 0.93 \mathrm{a}$ & $20.42 \pm 0.51 \mathrm{de}$ & $1.32 \pm 0.04 \mathrm{abc}$ \\
\hline XD3OSM1ox-3 & $95.00 \pm 1.53 \mathrm{c}$ & $23.04 \pm 2.43 \mathrm{ab}$ & $85.00 \pm 0.22 \mathrm{a}$ & $21.79 \pm 1.05 \mathrm{~cd}$ & $1.35 \pm 0.04 \mathrm{ab}$ \\
\hline XD3OSM1ox-4 & $98.33 \pm 0.33 \mathrm{a}$ & $23.83 \pm 0.98 \mathrm{a}$ & $83.25 \pm 0.90 \mathrm{ab}$ & $24.67 \pm 1.02 \mathrm{a}$ & $1.27 \pm 0.04 \mathrm{bc}$ \\
\hline XD3OSM1ox-5 & $97.33 \pm 0.77 a b$ & $24.17 \pm 0.36 \mathrm{a}$ & $83.00 \pm 0.58 a b$ & $22.67 \pm 1.67 \mathrm{bc}$ & $1.33 \pm 0.02 \mathrm{ab}$ \\
\hline XD3OSM1ox-6 & $97.07 \pm 0.33 \mathrm{ab}$ & $22.08 \pm 0.67 \mathrm{ab}$ & $83.25 \pm 0.66 \mathrm{ab}$ & $20.42 \pm 1.08 \mathrm{de}$ & $1.28 \pm 0.01 \mathrm{bc}$ \\
\hline XD3OSM1ox-7 & $97.00 \pm 0.00 \mathrm{ab}$ & $21.33 \pm 0.46 \mathrm{ab}$ & $79.75 \pm 1.04 \mathrm{c}$ & $19.08 \pm 0.55 \mathrm{e}$ & $1.30 \pm 0.03 \mathrm{abc}$ \\
\hline XD3OSM1ox-8 & $98.00 \pm 0.00 \mathrm{a}$ & $20.46 \pm 1.34 b$ & $80.08 \pm 1.33 \mathrm{c}$ & $20.96 \pm 0.51 \mathrm{cde}$ & $1.39 \pm 0.08 \mathrm{a}$ \\
\hline XD3OSM1ox-9 & $98.33 \pm 0.33 \mathrm{a}$ & $21.92 \pm 0.80 \mathrm{ab}$ & $79.17 \pm 1.67 \mathrm{c}$ & $22.50 \pm 1.04 \mathrm{bc}$ & $1.28 \pm 0.05 \mathrm{abc}$ \\
\hline$F / P$ & $4.242 / 0.029$ & $1.147 / 0.3786$ & $8.797 / 0.000$ & $6.219 / 0.0003$ & $1.644 / 0.1649$ \\
\hline
\end{tabular}

${ }^{\mathrm{z}} \mathrm{HD}=$ heading date (day of year), TA = Tiller angle, $\mathrm{PH}=$ plant height, FLH = flag leaf height, FLL = flag leaf length, and FLW = flag leaf width. Lowercase

letters indicate a $5 \%$ level or lower of statistic significance. Values with the same letter indicate no statistically significant difference.

Table 3. Productivity traits of partial transgenic lines and the wild-type (WT) control XD3 \pm standard deviation (SD) ${ }^{\mathrm{z}}$

\begin{tabular}{lclllcccc}
\hline Line & $\mathbf{P L} \pm \mathbf{S D}(\mathbf{c m})$ & $\mathbf{G L} \pm \mathbf{S D}(\mathbf{c m})$ & $\mathbf{G W} \pm \mathbf{S D}(\mathbf{c m})$ & $\mathbf{P N P} \pm \mathbf{S D}$ & $\mathbf{S N P} \pm \mathbf{S D}$ & $\mathbf{R F G} \pm \mathbf{S D}(\%)$ & $\mathbf{1 , 0 0 0 G W} \pm \mathbf{S D}(\mathbf{g})$ & YP $\pm \mathbf{S D}(\mathbf{g})$ \\
\hline WT & $16.96 \pm 0.04 \mathrm{a}$ & $0.71 \pm 0.009 \mathrm{c}$ & $0.34 \pm 0.009 \mathrm{a}$ & $8.45 \pm 0.18 \mathrm{a}$ & $152.66 \pm 5.18 \mathrm{a}$ & $89.44 \pm 1.08 \mathrm{a}$ & $25.82 \pm 0.09 \mathrm{ab}$ & $29.73 \pm 0.58 \mathrm{a}$ \\
XD3OSM1ox-1 & $17.33 \pm 0.18 \mathrm{a}$ & $0.73 \pm 0.003 \mathrm{bc}$ & $0.33 \pm 0.006 \mathrm{a}$ & $8.52 \pm 0.27 \mathrm{a}$ & $152.61 \pm 6.06 \mathrm{a}$ & $90.44 \pm 2.02 \mathrm{a}$ & $24.89 \pm 0.46 \mathrm{~b}$ & $29.24 \pm 1.54 \mathrm{a}$ \\
XD3OSM1ox-2 & $17.32 \pm 0.37 \mathrm{a}$ & $0.73 \pm 0.003 \mathrm{bc}$ & $0.33 \pm 0.007 \mathrm{a}$ & $7.98 \pm 0.24 \mathrm{a}$ & $153.54 \pm 3.77 \mathrm{a}$ & $90.35 \pm 0.71 \mathrm{a}$ & $25.98 \pm 0.79 \mathrm{ab}$ & $28.70 \pm 0.98 \mathrm{a}$ \\
XD3OSM10x-3 & $17.38 \pm 0.31 \mathrm{a}$ & $0.73 \pm 0.000 \mathrm{bc}$ & $0.33 \pm 0.008 \mathrm{a}$ & $8.27 \pm 0.52 \mathrm{a}$ & $155.33 \pm 6.01 \mathrm{a}$ & $89.87 \pm 0.83 \mathrm{a}$ & $25.40 \pm 0.40 \mathrm{ab}$ & $29.36 \pm 2.54 \mathrm{a}$ \\
XD3OSM1ox-5 & $16.58 \pm 0.79 \mathrm{a}$ & $0.74 \pm 0.014 \mathrm{~b}$ & $0.33 \pm 0.007 \mathrm{a}$ & $8.72 \pm 0.51 \mathrm{a}$ & $151.02 \pm 6.65 \mathrm{a}$ & $90.04 \pm 0.78 \mathrm{a}$ & $26.75 \pm 0.60 \mathrm{a}$ & $31.60 \pm 1.59 \mathrm{a}$ \\
XD3OSM10x-6 & $16.17 \pm 0.96 \mathrm{a}$ & $0.77 \pm 0.007 \mathrm{a}$ & $0.34 \pm 0.005 \mathrm{a}$ & $8.39 \pm 0.34 \mathrm{a}$ & $150.35 \pm 3.02 \mathrm{a}$ & $90.47 \pm 0.54 \mathrm{a}$ & $26.60 \pm 0.08 \mathrm{a}$ & $30.36 \pm 1.31 \mathrm{a}$ \\
$F / P$ & $0.776 / 0.5889$ & $6.791 / 0.0052$ & $1.531 / 0.289$ & $0.813 / 0.5668$ & $0.155 / 0.9735$ & $0.191 / 0.9593$ & $1.935 / 0.1751$ & $0.394 / 0.842$ \\
\hline
\end{tabular}

${ }^{\mathrm{z}} \mathrm{PL}=$ panicle length, $\mathrm{GL}=$ grain length, $\mathrm{GW}=$ grain width, $\mathrm{PNP}=$ panicle number per plant, $\mathrm{SNP}=$ seed number per panicle, $\mathrm{RFG}=$ ratio of filled grains, $1,000 \mathrm{GW}=1,000$-grain weight, and PY $=$ plant yield. Lowercase letters indicate a $5 \%$ level or lower of statistic significance. Values with the same letter indicate no statistically significant difference. 

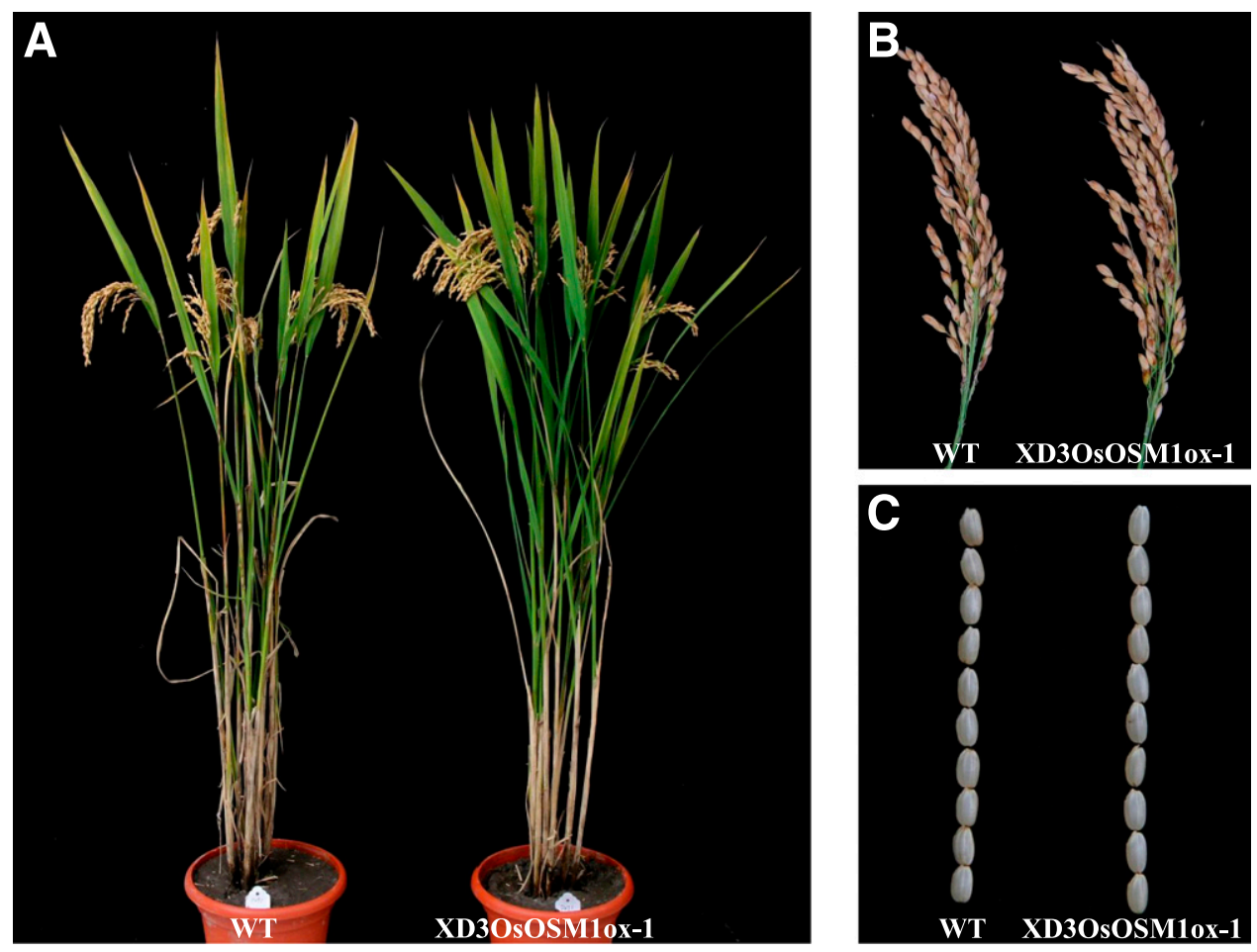

Fig. 4. Traits of XD3 OsOSM10x-1 transgenic plants. Representative pictures of whole plants, panicles, and seed of XD3 OsOSM10X-1 and wild type were shown. A, Plants were photographed at 30 days after heading. Photographs of B, panicles and $\mathbf{C}$, seed were taken at 60 days after heading.

A

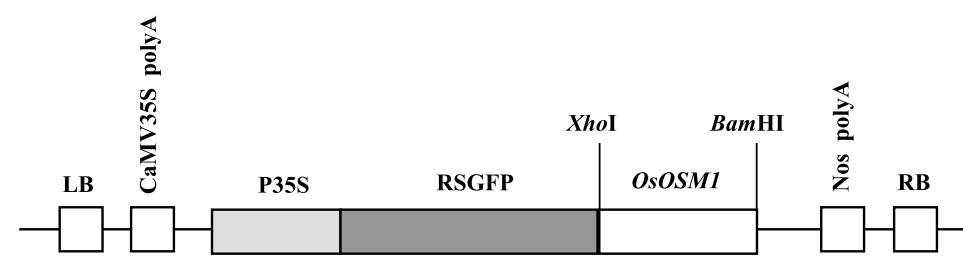

B
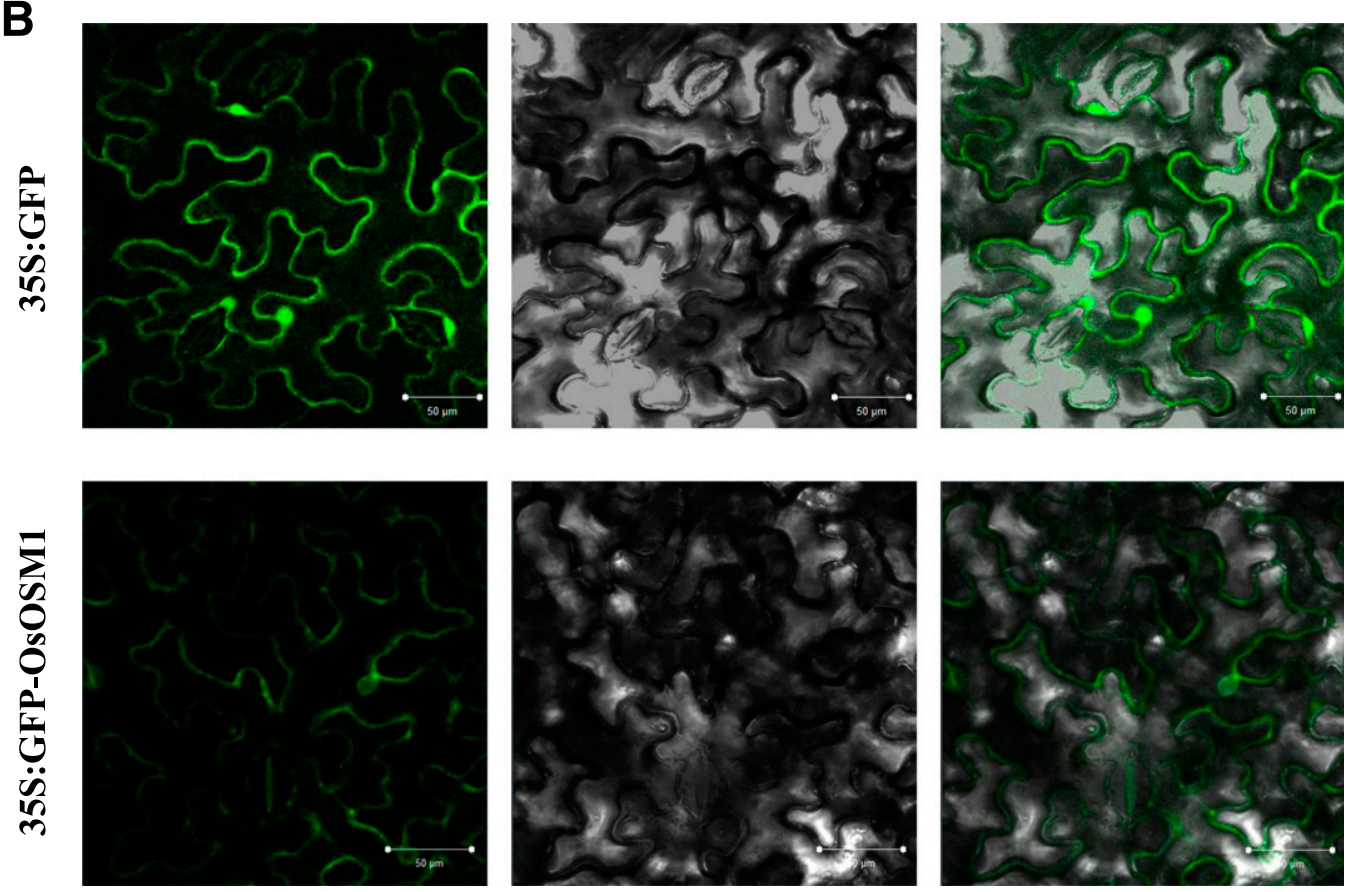

Fig. 5. Subcellular localization of the green fluorescent protein (GFP)-OsOSM1 fusion protein in tobacco leaf epidermal cells. A, Structure of the fusion protein construct used in transient expression. RSGFP = red-shifted GFP. B, Subcellular localization of GFP alone and the GFP-OsOSM1 fusion protein in tobacco leaf epidermal cells. Micrographs show cells expressing GFP (control, upper panel) or the GFP-OsOSM1 fusion protein (bottom panel), which was examined under fluorescent-field illumination (left) and bright-field illumination (middle), and an overlay of bright and fluorescent illumination (right). Bars $=50 \mu \mathrm{m}$. 
spatial expression patterns, we found that $O s O S M 1$ displays the highest expression level in the leaf sheath and panicle whereas OsOSM2 is mainly expressed in roots and stems (Fig. 2B). The fact that OsOSM1 is expressed highest in sheaths at the booting stage coincides with where and when the SB fungus infects rice in the field.

We then tested expression levels by quantitative (q)RT-PCR for both osmotin genes in response to $R$. solani infection in the resistant variety YSBR1 and two susceptible varieties, XD3 and Lemont. We found that, in YSBR1, the OSOSM1 level increases dramatically $12 \mathrm{~h}$ postinoculation and increases continuously through $72 \mathrm{~h}$ (Fig. 2C), whereas its expression was only slightly increased in the two SBsusceptible varieties. For OSM2, however, we did not find that its expression increased after $R$. solani inoculation (data not shown).

Taken together, these data suggest that the OsOSM1 gene may play an important role in defense against SB.

Overexpression of $\mathrm{OSOSM1}$ enhances rice resistance to SB. In order to evaluate the effect of $O S O S M 1$ on resistance to rice $\mathrm{SB}$, we cloned the $O S O S M 1$ gene and placed it under control of the maize ubiquitin-1 promoter to generate the OSOSM1ox construct p1301/ Ubi-OsOSM1 (Fig. 3A). Through Agrobacterium-mediated transformation, we introduced the OsOSMIox construct into the SB-susceptible $\mathrm{XD} 3$ and obtained nine independent $\mathrm{T}_{0}$ transgenic lines. The homozygous transgenic plants for each transgenic line were obtained in the $\mathrm{T}_{2}$ generation via PCR-based genotyping. The OsOSM1 mRNA expression levels in transgenic lines were determined by qRT-PCR. Compared with the WT (Fig. 3B), the OsOSMI expression level in each of the nine transgenic lines is significantly increased, reaching fivefold of the control in four lines (numbers 6, 7, 8, and 9). These results indicate that the OsOSM1 transgene is overexpressed in most transgenic lines.

We next tested SB resistance of these OsOSM1ox homozygous lines in the field. All lines were inoculated with the SB fungus at the tillering stage and scored at the mature stage. The SB scores of the transgenic lines ranged from 5.41 to 6.47 , all significantly lower than that of the WT (7.01) (Fig. 3C and 3D). In general, the OsOSM1 level was found inversely correlated with the SB score (Fig. 3E), even though the highest-expression line (number 8) was not the most resistant line, giving an SB score of 5.93. In summary, these results demonstrate that overexpression of $O S O S M 1$ is able to increase rice resistance to SB in the field.

Appropriate OsOSM1 overexpression level has no inferior effects on rice development or grain yield. To further assess the application potential of $O S O S M 1$ in rice breeding, we measured major agronomic traits of the OsOSM1ox transgenic lines and the WT control. We observed some statistically significant differences between four transgenic lines (numbers 4, 7, 8, and 9) and the WT on heading date or other morphological traits (Table 2). Most noticeably, the three transgenic lines carrying the highest $O S O S M 1$ expression levels (lines number 7, 8, and 9) all showed significantly lower plant height
( 80 versus $85 \mathrm{~cm}$ for the WT; Table 2). Although line 4 showed longer flag leaf length, it was not linked to the OsOSM1ox transgene because its null segregants also showed the longer flag leaf trait (data not shown). For the remaining five lines (numbers 1, 2, 3, 5, and 6), we observed no apparent alterations on the major agronomic traits compared with the WT. In general, the alternation of plant major agronomic traits would lead to the change of plant productivity traits. Therefore, we then harvested panicles and seed from the remaining five lines and the control and measured yield-associated components and seed traits (Table 3). Among the eight traits measured, we only found significant differences in grain length between transgenic lines 5 and 6 and the control; lines 5 and 6 displayed greater grain length ( 0.74 to 0.77 versus $0.71 \mathrm{~cm}$ for the WT; Table 3 ). The remaining three lines (numbers 1,2, and 3) shared almost identical yield performance and seed shape with the control (Fig. 4). The four lines (numbers 6, 7, 8, and 9) with the highest OsOSMlexpression levels all displayed alterations on either morphological or yield-associated traits; in contrast, the transgenic lines expressing three to five times OsOSM1 levels compared with the control exhibited no significant changes on plant morphology or grain yield. Taken together, we conclude that increasing OSOSMI expression to appropriate high levels (three- to fivefold) does not affect rice development or yield performance.

OsOSM1 localizes on membranes. To investigate the subcellular localization of the OsOSM1 protein, we generated a transient expression construct to fuse the OsOSM1 protein to GFP (Fig. 5A). The $O S O S M 1$ protein is a predicted membrane protein containing a transmembrane domain at amino acids 7 to 29 . When transiently expressed in $N$. benthamiana, we found that the GFP-OsOSM1 fusion protein is mainly localized on membranes, whereas the control GFP signal is present in whole cell (Fig. 5B). These results confirm that the OsOSM1 protein is a membrane-localized protein.

OsOSMIox activates defense genes associated with JA signaling pathway. JA- and ET-mediated defense pathways have been found involved in rice resistance to $\mathrm{SB}$; therefore, we analyzed the mRNA level of OsOSM1 after treatment with four defense-related hormones (JA, ET, SA, and KT). The effect of each of the four hormones was confirmed by monitoring the induction of their individual marker gene (Supplementary Fig. S1). We then assessed the OsOSM1 level and found that it was greatly elevated 1 day after JA treatment, peaking at day 2 before subsiding. In contrast, we observed no obvious induction after treatment with ET, SA, and KT individually (Fig. 6A). We further treated plants with JA inhibitor and found that the OsOSM1 level quickly decreased at days 1 and 2 but returned to normal at day 3 (Fig. 6A). These results indicate that $O S O S M 1$ may function in a JA-mediated signaling pathway.

In order to investigate the mechanism of enhanced resistance of OsOSM1ox lines to SB, the expression levels of three JA-associated defense marker genes were assessed using three transgenic lines
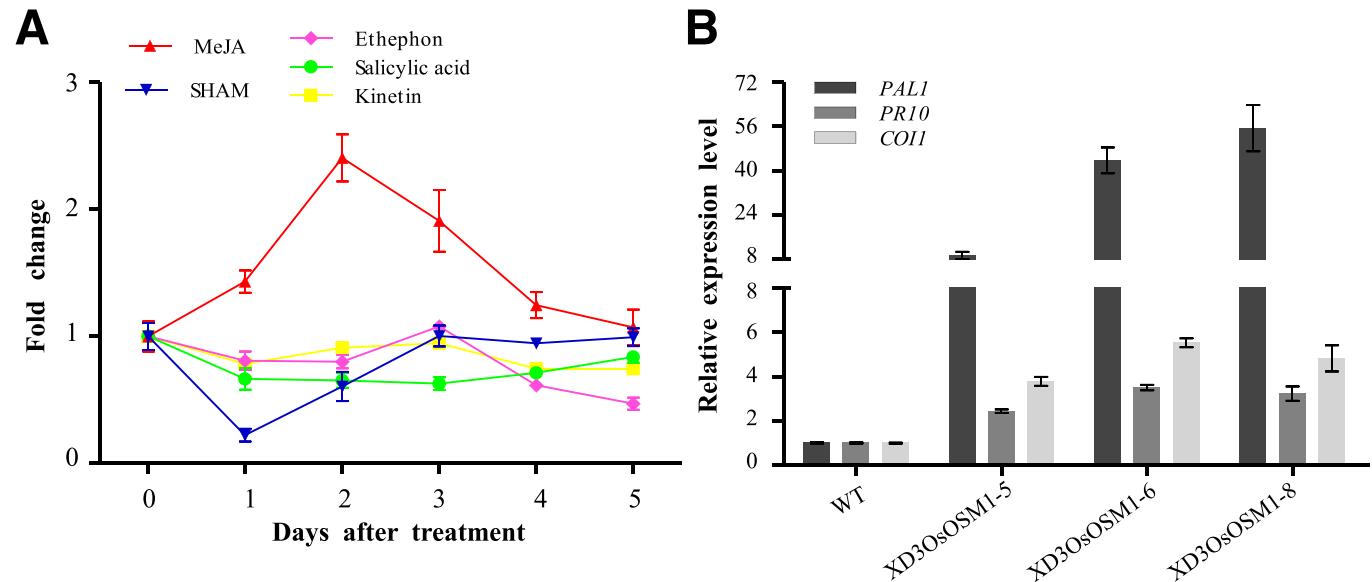

Fig. 6. Expression of OsOSM1 in response to different hormones and induction of jasmonic acid (JA)-responsive genes in XD3 OsOSM1ox transgenic lines. A, Expression of OsOSM1 in response to JA, salicylhydroxamic acid (SHAM), ethephon, salicylic acid, and kinetin treatments in 4-week-old seedlings. B, Induction of JA-responsive genes in OsOSM10x transgenic rice. Total RNA was extracted from the leaf sheath, and the $\beta$-actin gene was used as the internal control. 
(OsOSMlox lines 5, 6, and 8) with low, medium, and high expression levels of OsOSM1 (Fig. 6B). We found that the transcript levels of $P A L 1, P R 10$, and COII were all significantly elevated in these transgenic lines compared with the WT. Moreover, the transcript levels of the three marker genes were the lowest in line 5, coinciding with the lowest level of OSOSM1 in line 5. Taken together, we conclude that the enhanced SB resistance in the OSOSM1ox lines is associated with the activation of JA-associated defense genes such as PAL1, PR1O, and COII.

\section{Discussion}

Due to the complex nature of rice SB resistance, it has been very difficult to develop resistant varieties relying only on SBR QTL. To date, there are no more than 10 confirmed SBR QTL with observable contributions to SB resistance in field tests, even though extensive efforts have been made on mining SBR QTL. The $q S B-9$ locus has probably attracted more attention than other SBR QTL reported thus far (Liu et al. 2013; Srinivasachary et al. 2011; Taguchi-Shiobara et al. 2013; Zuo et al. 2008, 2014b) because it has been reported in several independent studies and confirmed to reduce the SB score by approximately 0.8 (0-to- 9 rating scale) in field tests using nearisogenic lines (Chen et al. 2014; Liu et al. 2013; Zuo et al. 2014a). All other SBR QTL were reported with either comparable or smaller effects than $q S B-9$ on improving SB resistance. In addition, complex interactions among these SBR QTL were reported (Chen et al. 2014), increasing the difficulty in pyramiding SBR QTL to improve rice SB resistance.

In this study, we found that overexpression of OsOSM1 is able to increase rice $\mathrm{SB}$ resistance by reducing SB scores ranging from 0.54 to 1.59 (0-to-9 rating scale; Fig. 3). However, we also noted that lines with the highest OsOSM1 levels displayed apparent alterations in plant morphology, whereas lines with moderately higher levels (three- to fivefold) showed no observable changes in plant morphology (Table 2). Therefore, we believe that an increase in OsOSM1 expression to appropriate levels is capable of improving SB resistance without affecting rice development and grain yield (Fig. 3; Tables 2 and 3). Two of nine (OsOSM1ox lines 1 and 3) transgenic lines carried $O s O S M 1$ expression levels three to five times higher than the WT and showed morphology and yield performance identical to the WT. The average SB score of these two lines was 6.12, effectively reducing the SB score by 0.89 . These results demonstrate that the effect of overexpressing an appropriate amount of OsOSM1 is comparable with the effects of major SBR QTL identified thus far. Consequently, we believe that the OSOSM1 gene has great potential against SB disease in rice breeding.

Using qRT-PCR, we found that OsOSM1 is highly expressed at the booting developmental stage and mainly in the rice sheath. Also, OsOSM1 was quickly induced by SB fungal infection in an SB-resistant variety but not in two susceptible varieties. Overexpression of $O S O S M 1$ in one of the susceptible varieties significantly enhanced SB resistance. These results indicate that OsOSM1 plays an important role in defense against SB disease. Although OsOSM2 shares the highest identity with OSOSM1, it presents a totally different gene expression pattern, with the highest levels in roots and stems, implying that it is probably involved in rice resistance to root and stem diseases. In order to investigate the resistant mechanism of OsOSM1, we determined its subcellular localization to cell membranes using a tobacco transient expression system. We also found that $O S O S M 1$ expression was induced by JA treatment and that the treatment of a JA inhibitor downregulated OSOSM1 expression (Fig. 6A). Generally, the JA- and ET-mediated signaling pathways are considered to work synergistically in defense response to necrotrophic pathogens such as $R$. solani; in contrast, the SA signal is associated with defense against biotrophic pathogens (Glazebrook 2005). Recently, increasing studies have emphasized the importance of cytokine (CK)-mediated signaling in regulation of both plant disease resistance and grain yield (Naseem et al. 2014). However, CK treatment did not induce OSOSM1 expression. These data allowed us to conclude that OSOSMI functions in the JA signaling pathway. Consistent with this, we found JA-associated defense genes upregulated in the OsOSMIox lines. Consequently, we hypothesize that the increased resistance to $\mathrm{SB}$ in the OsOSMlox lines is dependent on the activation of JA defense signaling.

Genome editing, which modifies endogenous genes, has garnered much popularity (Gaj et al. 2013) and may pose fewer safety issues than traditional transformation technology (Schaeffer and Nakata 2015). Identifying the suitable targets for genome editing is an essential step in improving rice SB resistance. Thus far, only a few genes, such as OsPGIP1, OsACS2, tlp-D34, chill, and RCH10, were shown to improve rice SB resistance in the field (Chen et al. 2015; Datta et al. 2001; Helliwell et al. 2013; Mao et al. 2014; Shah et al. 2013; Wang et al. 2015). In this study, we found that appropriate overexpression of OSOSM1 is able to increase rice SB resistance without affecting rice development and grain yield. Thus, OsOSMI may serve as a new target for genome editing in the future to enhance rice resistance to $\mathrm{SB}$.

\section{Acknowledgments}

This work was partially supported by the key project of Jiangsu Natural Science Foundation for Colleges (14KJA210003), the National Transgenic key Project (2014ZX0800103B), the innovation project for graduate student in Jiangsu province (CXLX13_913), and a project funded by the Priority Academic Program Development of Jiangsu Higher Education Institutions.

\section{Literature Cited}

Anžlovar, S., and Dermastia, M. 2003. The comparative analysis of osmotins and osmotin-like PR-5 proteins. Plant Biol. 5:116-124.

Chen, X. J., Chen, Y., Zhang, L. N., Xu, B., Zhang, J. H., Chen, Z. X., Tong, Y. H., Zuo, S. M., and Xu, J. Y. 2015. Overexpression of OsPGIP1 enhances rice resistance to sheath blight. Plant Dis. 100:388-395.

Chen, Z. X., Zhang, Y. F., Feng, F., Feng, M. H., Jiang, W., Ma, Y. Y., Pan, C. H., Hua, H. L., Li, G. S., and Pan, X. B. 2014. Improvement of japonica rice resistance to sheath blight by pyramiding $q S B-9^{T Q}$ and $q S B-7^{T Q}$. Field Crops Res. 161:118-127.

Choi, D. S., Hong, J. K., and Hwang, B. K. 2013. Pepper osmotin-like protein 1 (CaOSM1) is an essential component for defense response, cell death, and oxidative burst in plants. Planta 238:1113-1124.

Datta, K., Tu, J., Oliva, N., Ona, I., Velazhahan, R., Mew, T. W., Muthukrishnan, S., and Datta, S. K. 2001. Enhanced resistance to sheath blight by constitutive expression of infection-related rice chitinase in transgenic elite indica rice cultivars. Plant Sci. 160:405-414.

Datta, K., Velazhahan, R., Oliva, N., Ona, I., Mew, T., Khush, G., Muthukrishnan, S., and Datta, S. 1999. Over-expression of the cloned rice thaumatin-like protein (PR-5) gene in transgenic rice plants enhances environmental friendly resistance to Rhizoctonia solani causing sheath blight disease. Theor. Appl. Genet. 98:1138-1145.

De Lorenzo, G., D'Ovidio, R., and Cervone, F. 2001. The role of polygalacturonaseinhibiting proteins (PGIPs) in defense against pathogenic fungi. Annu. Rev. Phytopathol. 39:313-335.

De Vleesschauwer, D. 2008. Unraveling rhizobacteria- and abscisic acid-induced pathogen resistance in rice (Oryza sativa L.). Ph.D. thesis, Ghent University, Belgium.

De Vleesschauwer, D., Yang, Y., Cruz, C. V., and Höfte, M. 2010. Abscisic acidinduced resistance against the brown spot pathogen Cochliobolus miyabeanus in rice involves MAP kinase-mediated repression of ethylene signaling. Plant Physiol. 152:2036-2052.

Gaj, T., Gersbach, C. A., and Barbas, C. F. 2013. ZFN, TALEN, and CRISPR/Casbased methods for genome engineering. Trends Biotechnol. 31:397-405.

Glazebrook, J. 2005. Contrasting mechanisms of defense against biotrophic and necrotrophic pathogens. Annu. Rev. Phytopathol. 43:205-227.

Goodin, M. M., Dietzgen, R. G., Schichnes, D., Ruzin, S., and Jackson, A. O 2002. pGD vectors: Versatile tools for the expression of green and red fluorescent protein fusions in agroinfiltrated plant leaves. Plant J. 31:375-383.

Helliwell, E. E., Wang, Q., and Yang, Y. 2013. Transgenic rice with inducible ethylene production exhibits broad-spectrum disease resistance to the fungal pathogens Magnaporthe oryzae and Rhizoctonia solani. Plant Biotechnol. J. 11:33-42.

Hiei, Y., Ohta, S., Komari, T., and Kumashiro, T. 1994. Efficient transformation of rice (Oryza sativa L.) mediated by Agrobacterium and sequence analysis of the boundaries of the T-DNA. Plant J. 6:271-282.

Ito, Y., and Kurata, N. 2006. Identification and characterization of cytokininsignalling gene families in rice. Gene 382:57-65.

Jia, Y. L., Liu, G. J., Costanzo, S., Lee, S., and Dai, Y. T. 2009. Current progress on genetic interactions of rice with rice blast and sheath blight fungi. Front. Agric. China 3:231-239.

Kagale, S., Marimuthu, T., Kagale, J., Thayumanavan, B., and Samiyappan, R. 2011. Induction of systemic resistance in rice by leaf extracts of Zizyphus jujuba and Ipomoea carnea against Rhizoctonia solani. Plant Signal. Behav. 6:919-923.

Kok, E. J., Pedersen, J., Onori, R., Sowa, S., Schauzu, M., De Schrijver, A., and Teeri, T. H. 2014. Plants with stacked genetically modified events: To assess or not to assess? Trends Biotechnol. 32:70-73. 
Lee, F. N., and Rush, M. C. 1983. Rice sheath blight: A major rice disease. Plant Dis. 67:829-833.

Liu, D., Raghothama, K. G., Hasegawa, P. M., and Bressan, R. A. 1994. Osmotin overexpression in potato delays development of disease symptoms. Proc. Natl. Acad. Sci. USA 91:1888-1892.

Liu, G. J., Jia, Y. L., McClung, A., Oard, J. H., Lee, F. N., and Correll, J. C. 2013. Confirming QTLs and finding additional loci responsible for resistance to rice sheath blight disease. Plant Dis. 97:113-117.

Mao, B., Liu, X., Hu, D., and Li, D. 2014. Co-expression of RCH10 and AGLU1 confers rice resistance to fungal sheath blight Rhizoctonia solani and blast Magnaporthe oryzae and reveals impact on seed germination. World J. Microbiol. Biotechnol. 30:1229-1238.

Narasimhan, M. L., Coca, M. A., Jin, J., Yamauchi, T., Ito, Y., Kadowaki, T., Kim, K. K., Pardo, J. M., Damsz, B., and Hasegawa, P. M. 2005. Osmotin is a homolog of mammalian adiponectin and controls apoptosis in yeast through a homolog of mammalian adiponectin receptor. Mol. Cell 17:171-180.

Naseem, M., Wölfling, M., and Dandekar, T. 2014. Cytokinins for immunity beyond growth, galls and green islands. Trends Plant Sci. 19:481-484.

Penninckx, I., Eggermont, K., Terras, F., Thomma, B., De Samblanx, G. W., Buchala, A., Métraux, J. P., Manners, J. M., and Broekaert, W. F. 1996. Pathogen-induced systemic activation of a plant defensin gene in Arabidopsis follows a salicylic acid-independent pathway. Plant Cell 8:2309-2323.

Schaeffer, S. M., and Nakata, P. A. 2015. CRISPR/Cas9-mediated genome editing and gene replacement in plants: Transitioning from lab to field. Plant Sci. 240: 130-142.

Schultz, J., Milpetz, F., Bork, P., and Ponting, C. P. 1998. SMART, a simple modular architecture research tool: Identification of signaling domains. Proc. Natl. Acad. Sci. USA 95:5857-5864.

Shah, J. M., Singh, R., and Veluthambi, K. 2013. Transgenic rice lines constitutively co-expressing tlp-D34 and chill display enhancement of sheath blight resistance. Biol. Plant. 57:351-358.

Singh, N. K., Bracker, C. A., Hasegawa, P. M., Handa, A. K., Buckel, S., Hermodson, M. A., Pfankoch, E. D., Regnier, F. E., and Bressan, R. A. 1987. Characterization of osmotin A thaumatin-like protein associated with osmotic adaptation in plant cells. Plant Physiol. 85:529-536.

Taguchi-Shiobara, F., Ozaki, H., Sato, H., Maeda, H., Kojima, Y., Ebitani, T., and Yano, M. 2013. Mapping and validation of QTLs for rice sheath blight resistance. Breed. Sci. 63:301-308.

Tamura, K., Stecher, G., Peterson, D., Filipski, A., and Kumar, S. 2013. MEGA6: Molecular evolutionary genetics analysis version 6.0. Mol. Biol. Evol. 30: 2725-2729.

Wang, R., Lu, L., Pan, X., Hu, Z., Ling, F., Yan, Y., Liu, Y., and Lin, Y. 2015. Functional analysis of $O S P G I P 1$ in rice sheath blight resistance. Plant Mol. Biol. 87:181-191.
Srinivasachary, Willocquet, L., and Savary, S. 2011. Resistance to rice sheath blight (Rhizoctonia solani Kühn)[(teleomorph: Thanatephorus cucumeris (AB Frank) Donk.] disease: Current status and perspectives. Euphytica 178:1-22.

Xie, D. X., Feys, B. F., James, S., Nieto-Rostro, M., and Turner, J. G. 1998. COI1 An Arabidopsis gene required for jasmonate-regulated defense and fertility. Science 280:1091-1094.

Xu, Q., Yuan, X. P., Yu, H. Y., Wang, Y. P., Tang, S. X., and Wei, X. H. 2011. Mapping quantitative trait loci for sheath blight resistance in rice using double haploid population. Plant Breed. 130:404-406.

Yin, Y. J. 2008. Genetic analysis and fine mapping of a QTL $q S B-9^{T q}$ conferring resistance to sheath blight in rice. Ph.D. thesis, Yangzhou University, Yangzhou, China.

Zhu, Y. J., Zuo, S. M., Chen, Z. X., Chen, X. G., Li, G., Zhang, Y. F., Zhang, G. Q., and Pan, X. B. 2014. Identification of two major rice sheath blight resistance QTLs, $q S B 1-1^{H J X 74}$ and $q S B 11^{H J X 74}$, in field trials using chromosome segment substitution lines. Plant Dis. 98:1112-1121.

Zou, J. H., Pan, X. B., Chen, Z. X., Xu, J. Y., Lu, J. F., Zhai, W. X., and Zhu, L. H. 2000. Mapping quantitative trait loci controlling sheath blight resistance in two rice cultivars (Oryza sativa L.). Theor. Appl. Genet. 101:569-573.

Zuo, S. M., Wang, Z. B., Chen, X. J., Gu, F., Zhang, Y. F., Chen, Z. X., and Pan, X. B. 2009. Evaluation of resistance of a novel rice germplasm YSBR1 to sheath blight. Acta Agron. Sin. 35:608-614.

Zuo, S. M., Yin, Y. J., Pan, C. H., Chen, Z. X., Zhang, Y. F., Gu, S. L., Zhu, L. H., and Pan, X. B. 2013. Fine mapping of $q S B-11^{L E}$, the QTL that confers partial resistance to rice sheath blight. Theor. Appl. Genet. 126:12571272 .

Zuo, S. M., Zhang, L., Wang, H., Yin, Y. J., Zhang, Y. F., Chen, Z. X., Ma, Y. Y., and Pan, X. B. 2008. Prospect of the QTL-qSB-9 ${ }^{\text {Tq }}$ utilized in molecular breeding program of japonica rice against sheath blight. J. Genet. Genomics 35:499-505.

Zuo, S. M., Zhang, Y. F., Chen, Z. X., Chen, X. J., and Pan, X. B. 2010. Current progress on genetics and breeding in resistance to rice sheath blight. Sci. Sin Vitae 40:1014-1023.

Zuo, S. M., Zhang, Y. F., Yin, Y. J., Chen, Z. X., and Pan, X. B. 2006 Establishment and improvement of inoculation technique and rating system in researching rice sheath blight resistance in field. J. Yangzhou Univ. 27: 57-61. (Agricultural and Life Science Edition)

Zuo, S. M., Zhang, Y. F., Yin, Y. J., Li, G. Z., Zhang, G. W., Wang, H., Chen, Z. X., and Pan, X. B. 2014a. Fine-mapping of $q S B-9^{T Q}$, a gene conferring major quantitative resistance to rice sheath blight. Mol. Breed. 34:2191-2203.

Zuo, S. M., Zhu, Y. J., Yin, Y. J., Wang, H., Zhang, Y. F., Chen, Z. X., Gu, S. L., and Pan, X. B. 2014b. Comparison and confirmation of quantitative trait loci conferring partial resistance to rice sheath blight on chromosome 9. Plant Dis. 98:957-964. 